\title{
Influence of deprivation on health care use, health care costs, and mortality in COPD
}

This article was published in the following Dove Press journal:

International Journal of COPD

\author{
Peter F Collins ${ }^{1-3}$ \\ Rebecca J Stratton' \\ Ramesh J Kurukulaaratchy ${ }^{4,5}$ \\ Marinos Elia' \\ 'NIHR Nutrition Biomedical \\ Research Centre, Faculty of \\ Medicine, University of Southampton, \\ Southampton General Hospital, \\ University Hospital Southampton \\ NHS Foundation Trust, Southampton, \\ Hampshire, UK; ${ }^{2}$ Nutrition and \\ Dietetics, School of Exercise and \\ Nutrition Sciences, Faculty of Health, \\ Queensland University of Technology, \\ Brisbane, QLD, Australia; ${ }^{3}$ Department \\ of Nutrition and Dietetics, Princess \\ Alexandra Hospital, Brisbane, \\ QLD, Australia; ${ }^{4} \mathrm{NIHR}$ Respiratory \\ Biomedical Research Centre, \\ Southampton General Hospital, \\ University Hospital Southampton \\ NHS Foundation Trust, Southampton, \\ Hampshire, UK; ${ }^{5} \mathrm{Clinical}$ and \\ Experimental Sciences, Faculty of \\ Medicine, University of Southampton, \\ Southampton, Hampshire, UK
}

Correspondence: Peter F Collins Nutrition and Dietetics, School of Exercise and Nutrition Sciences, Faculty of Health, Queensland University of Technology, Victoria Park Road, Brisbane, QLD 4059, Australia

Tel +6I 731383524

Email pf.collins@qut.edu.au
Background and aim: Deprivation is associated with the incidence of COPD, but its independent impact on clinical outcomes is still relatively unknown. This study aimed to explore the influence of deprivation on health care use, costs, and survival.

Methods: A total of 424 outpatients with COPD were assessed for deprivation across two hospitals. The English Index of Multiple Deprivation (IMD) was used to establish a deprivation score for each patient. The relationship between deprivation and 1-year health care use, costs, and mortality was examined, controlling for potential confounding variables (age, malnutrition risk, COPD severity, and smoking status).

Results: IMD was significantly and independently associated with emergency hospitalization ( $\beta$-coefficient 0.022 , SE $0.007 ; p=0.001$ ), length of hospital stay, secondary health care costs ( $\beta$-coefficient $£ 101, \mathrm{SE} £ 30 ; p=0.001$ ), and mortality (HR 1.042, 95\% CI 1.015-1.070; $p=0.002$ ). IMD was inversely related to participation in exercise rehabilitation (OR $0.961,95 \% \mathrm{CI}$ $0.930-0.994 ; p=0.002$ ) and secondary care appointments. Deprivation was also significantly related to modifiable risk factors (smoking status and malnutrition risk).

Conclusion: Deprivation in patients with COPD is associated with increased emergency health care use, health care costs, and mortality. Tackling deprivation is complex; however, strategies targeting high-risk groups and modifiable risk factors, such as malnutrition and smoking, could reduce the clinical and economic burden.

Keywords: COPD, economics, socioeconomic, deprivation

\section{Introduction}

National and international policies aim to tackle health inequalities associated with deprivation, yet the health gradient between affluent and more-deprived communities continues to be of concern, with incidence rates for diseases such as COPD being significantly higher in deprived areas. ${ }^{1}$ The milestone Black Report published by the UK Government ${ }^{2}$ highlighted the need to address the large differences in morbidity and mortality that existed between social classes. Yet, a decade later, differences in mortality were continuing to grow, ${ }^{3}$ and some 30 years after the initial Black Report, more individuals in deprived areas were living shorter lives of poorer quality. ${ }^{4}$ More recently, the Marmot Review highlighted the enormous impact of health inequalities on life expectancy, with 2.5 million years of life potentially lost in England each year through premature death due to health inequalities, with people from the poorest neighborhoods dying - on average -7 years sooner. ${ }^{5}$

The socioeconomic gradient in COPD is as great, if not greater, than any other disease. ${ }^{6}$ The clustering of deprivation and COPD has been highlighted, whereby the majority of areas classified as having populations at high risk of COPD in the UK were in northern England and Scotland, ${ }^{7}$ with $98 \%$ of the most deprived areas being urban. ${ }^{8}$ 
Social inequalities can be associated with increased rates of respiratory infections requiring hospitalization, ${ }^{9}$ and increased mortality from COPD may be more likely to occur in urban, deprived areas. Research involving $>2.8$ million patients requiring emergency hospitalization across England over a period of 12 months found the Index of Multiple Deprivation (IMD) to be a significant predictor of hospitalization. ${ }^{10}$ COPD patients accounted for one of the highest annual emergency admission rates $(n=48,281)$ among all reported conditions, with $27 \%$ becoming "high-impact service users", defined as those who experience an emergency admission followed by a further two emergency admissions within 12 months. More recently, IMD has also been found to be independently associated with malnutrition risk in outpatients with COPD. ${ }^{11}$ IMD combines a number of indicators covering a range of economic, social, educational, and housing issues into a single deprivation score (IMD score). It has been applied to small areas (postcodes) throughout the UK, allowing comparison of deprivation closer to the individual level. Despite its utility, there are currently insufficient data specifically exploring the independent influence of deprivation on mortality and health care burden, including health care costs, in outpatients with COPD. Therefore, the aim of the current study was to explore whether deprivation was an independent determinant of poor clinical outcomes and health care costs in outpatients with COPD.

\section{Methods}

Consecutive outpatients, with a confirmed diagnosis of COPD (postbronchodilator forced expiratory volume in 1 second $\left[\mathrm{FEV}_{1}\right]$ of $<70 \%$ predicted and an $\mathrm{FEV}_{1} /$ forced vital capacity (FVC) ratio of $<70 \%$ ), attending outpatient respiratory clinics were routinely assessed for malnutrition risk as part of a larger randomized controlled trial, which also included assessment of deprivation. Outpatients $(n=424)$ were included across two hospital sites in Hampshire, England: a large, inner-city teaching hospital (site A: Southampton General Hospital); and a smaller, more-rural hospital in a more-affluent area within a National Park (site B: Lymington New Forest Hospital). Patients were assigned an IMD score on the basis of their address (postcode) at the time of their visit to the clinic. ${ }^{8}$ IMD allows an overall estimation of deprivation, as well as its seven component domains (income, employment, health deprivation/disability, education/training/skills, barriers to housing, crime, and living environment) for small residential areas (rather than for individual subjects). The higher the deprivation score for a particular postcode, the more likely an individual is to experience deprivation.
Demographic data were collected from electronic hospital records, and COPD severity was classified according to the Global Initiative for Management of Obstructive Lung Disease (GOLD) 2010 criteria. ${ }^{12}$ All patients were prospectively screened for risk of malnutrition using the Malnutrition Universal Screening Tool (MUST), ${ }^{13}$ and current smoking status was assessed. Health care use data, including admissionand-discharge dates and the nature of the admission (elective or emergency), were retrospectively collected 1 year after the date of assessment. It was not possible to obtain accurate data on the exact causes of the admissions and the associated disease severity from the electronic records, but clear data were obtained on respiratory outpatient clinic attendance and participation in exercise rehabilitation. Mortality data at 1 year after initial assessment were documented, and overall health care costs were estimated using the UK Department of Health National Health Service (NHS) bed stay costs ${ }^{14}$ and specific codes for COPD (eg, emergency admission/ ventilated or emergency admission/ambulatory).

All the statistical analyses were undertaken with SPSS statistical package, version 23.0 (IBM Corporation, Armonk, NY, USA). The tests used included analysis of variance (ANOVA), chi-squared analysis, multiple regression (general linear model - univariate analysis), binary logistic regression, and Cox regression, the final three being used to adjust for the following potential confounding variables (referred to as "confounders" in the article): age, COPD severity (moderate, severe, and very severe), smoking status (nonsmoker/exsmoker and current smoker), and malnutrition risk (medium risk and high risk). A $p$-value of $<0.05$ (two-tailed) was considered to be significant. Ethical approval for the study was granted by Southampton University Hospital NHS Foundation Trust (ELIA002) as part of a larger program of research that included a trial of nutritional support in COPD (ClinicalTrials.gov identifier NCT00538200). All data accessed for this observational study were anonymized, and, therefore, the Southampton University Hospital NHS Foundation Trust waived the need for informed consent. The study was guided by the Strengthening the Reporting of Observational Studies in Epidemiology (STROBE) recommendations.

\section{Results}

\section{General}

Baseline characteristics were not significantly different between the two hospital sites, with the exception of deprivation scores and malnutrition risk classification (Table 1). Despite being only 16 miles $(25.7 \mathrm{~km})$ apart, outpatients attending site A were significantly more likely to reside in 
Table I Baseline characteristics by hospital site

\begin{tabular}{|c|c|c|c|c|}
\hline Characteristics & $\begin{array}{l}\text { Site A } \\
(n=190)\end{array}$ & $\begin{array}{l}\text { Site B } \\
(n=234)\end{array}$ & $\begin{array}{l}\text { Total } \\
(n=424)\end{array}$ & $p$-value \\
\hline Females:males & 93:97 & $109: 125$ & $202: 222$ & 0.628 \\
\hline Age (years) & $72(10)$ & $74(10)$ & $73(10)$ & 0.078 \\
\hline COPD severity ${ }^{12}$ & $n=166$ & $n=223$ & $n=389$ & \\
\hline Moderate (II) & 46 (27.7\%) & $53(23.8 \%)$ & 99 (25.4\%) & - \\
\hline Severe (III) & $62(37.3 \%)$ & 97 (43.5\%) & 159 (40.9\%) & - \\
\hline Very severe (IV) & 58 (34.9\%) & 73 (32.7\%) & |3| (33.7\%) & 0.450 \\
\hline Smoking status & $n=186$ & $n=233$ & $\mathrm{n}=419$ & - \\
\hline Never & $5(2.7 \%)$ & $13(5.6 \%)$ & I8 (4.3\%) & - \\
\hline Ex-smoker & I 44 (77.4\%) & $179(76.8 \%)$ & 323 (77.1\%) & - \\
\hline Current smoker & 37 (19.9\%) & $4 \mathrm{I}(17.6 \%)$ & 78 (I8.6\%) & 0.315 \\
\hline Anthropometry & $\mathrm{n}=186$ & $n=234$ & $n=420$ & - \\
\hline Weight $(\mathrm{kg})$ & $69.8(20.2)$ & $72.6(18.1)$ & $71.4(19.1)$ & 0.134 \\
\hline BMI $\left(\mathrm{kg} / \mathrm{m}^{2}\right)$ & $25.4(6.7)$ & $26.1(6.0)$ & $25.8(6.3)$ & 0.232 \\
\hline \multicolumn{5}{|l|}{ MUST risk cat ${ }^{2}, *$} \\
\hline Low & $136(72 \%)$ & 195 (83\%) & 331 (78\%) & - \\
\hline Medium + High & $54(28 \%)$ & $39(17 \%)$ & $93(22 \%)$ & 0.004 \\
\hline Deprivation & $n=190$ & $n=234$ & $\mathrm{n}=424$ & - \\
\hline IMD score & $21.25(12.4)$ & II.56 (7.4) & $15.90(11.1)$ & $<0.001$ \\
\hline
\end{tabular}

Notes: Continuous variables are presented as mean (SD) and analyzed using ANOVA; categorical variables are presented as mean (\%) and analyzed using chisquared analysis; site $\mathrm{A}=$ inner-city hospital; site $\mathrm{B}=$ community hospital; *MUST risk cat $^{2}=$ Low vs Medium + High "MUST" categories.

Abbreviations: BMI, body mass index; IMD, Index of Multiple Deprivation; MUST, Malnutrition Universal Screening Tool.

deprived areas, with IMD score ranging from 1.88 (least deprived) to 57.05 (most deprived).

Current smoking status was recorded for 419 outpatients, $78(18.3 \%)$ of whom reported to be current smokers. Current smokers were younger than never- or ex-smokers (mean age: 67 years [SD 10 years] vs 74 years [SD 9 years]; $p<0.001$ ) and more likely to be malnourished (medium/high risk: smokers $32.1 \%$ vs nonsmokers/ex-smokers $19.4 \%$; $p=0.014$ ). Smoking status was not related to the hospital site attended ( $p=0.549)$, COPD severity ( $p=0.895$ ), or 1 -year mortality ( $p=0.511$ ), but it was significantly associated with IMD score (smokers: 20.4 [SD 14.8]; vs nonsmoker/ex-smokers: 14.8 [SD 10.4]; $p<0.001)$ and several of its domains, with current smokers experiencing significantly more deprivation related to employment, health/disability, education, and crime. Similarly, malnutrition risk was significantly related to IMD score (medium/high malnutrition risk: 18.3 [SD 11.4] vs low malnutrition risk 15.2 [SD 10.9]; $p=0.018$ ) and several of its domains, with malnourished experiencing more deprivation due to health/disability, crime, and living environment.

COPD severity could only be confirmed in $92 \%$ of the cohort ( $\mathrm{n}=389$ ), with the remainder unable to be confirmed in the electronic medical records. COPD severity and deprivation were not related, but an inverse association was observed between age and deprivation (IMD score for people aged $<65$ years [ $n=90] 18.30$ [SD 11.66]; for those aged 65-74.9 years [n=135]: 16.60 [SD 11.71]; for those aged $>75$ years $[\mathrm{n}=199] 14.35$ [SD 10.10]; ANOVA $p=0.013, p$ for linear trend $=0.005$ ). This remained significant ( $p$ for linear trend $=0.033$ ) after adjustment for malnutrition risk, COPD severity, and smoking status. There was no significant difference in the age of patients attending the two sites (Table 1), but a large difference was found in the IMD scores (21.3 [SD 12.4] vs 11.6 [SD 7.4]; $p<0.001$ ), and, with the exception of one domain (barriers to housing), large differences existed for all of the IMD domains ( $p$-values $<0.001$ ).

Smoking status was not only strongly associated with deprivation but also with emergency hospitalization, since $44.9 \%$ of current smokers experienced an emergency admission compared to $29.3 \%$ of ex-smokers or never-smokers ( $p=0.008$ ). It was also related to the annual number of emergency hospital admissions (smokers 1.22 [SD 2.66] vs nonsmokers/ex-smokers 0.48 [SD 0.96]; $p<0.001$ ) and the duration of admissions (smokers 8.4 [SD 17.1] days vs nonsmokers/ex-smokers 4.2 [SD 12.1] days; $p=0.013$ ). COPD severity was also associated with emergency hospital admissions ( $\beta$-coefficient 0.23 , SE $0.10 ; p=0.017$ ) and their durations ( $\beta$-coefficient 2.17, SE $0.88 ; p=0.014$ ), but additionally, it was related to mortality (OR $1.679,95 \% \mathrm{CI}$ $1.052-2.679 ; p=0.030$ ). Malnutrition was also related to mortality (medium/high risk $19.4 \%$ vs low risk $8.2 \%$; $p=0.002$ ), as was age (HR $1.04495 \%$ CI 1.010-1.078; $p=0.007$ ). After adjustment for confounding variables, all these relationships remained significant, including IMD, but in addition, COPD severity also became significantly related to participation in exercise rehabilitation (OR 1.591, 95\% CI 1.064-2.379; $p=0.024$ [unadjusted $p=0.062$ ]), and age became significantly and inversely related to the number of secondary care appointments ( $\beta$-coefficient -0.056 , SE 0.023 ; $p=0.015$ [unadjusted $p=0.051$ ]). Age was not associated with health care use, but it was related to mortality (survivors 72 [SD 10] years vs nonsurvivors 78 [SD 9] years; $p=0.008$ ).

\section{Health care use}

A third of the cohort (32\%) had at least one emergency admission during the 12-month follow-up period. Outpatients requiring emergency hospitalization were more likely to reside in deprived areas (IMD score 18.12 [SD 12.64] vs 14.84 [SD 10.07]; $p=0.004)$. Analysis of health care use after adjustment for confounders revealed that deprivation was a significant and independent predictor of the number of emergency admissions and subsequent hospital length of stay (LOS) (Table 2). While IMD was not associated with the number of elective admissions, it was the only covariate 
Table 2 Influence of deprivation (IMD score) on I-year health care use $(n=384)$

\begin{tabular}{llll}
\hline $\begin{array}{l}\text { I-year health care use per } \\
\text { patient* }\end{array}$ & $\beta$-coefficient & SE & $p$-value \\
\hline $\begin{array}{l}\text { Number of emergency hospital } \\
\text { admissions }(n)^{\mathrm{a}}\end{array}$ & 0.022 & 0.007 & 0.001 \\
$\begin{array}{l}\text { Emergency length of hospital } \\
\text { stay (days) }\end{array}$ & 0.139 & 0.062 & 0.026 \\
$\begin{array}{l}\text { Number of visits to accident } \\
\text { and emergency department }(n)^{\mathrm{c}}\end{array}$ & 0.006 & 0.005 & 0.243 \\
$\begin{array}{l}\text { Number of elective hospital } \\
\text { admissions ( } \mathrm{n})^{\mathrm{d}}\end{array}$ & $-0.00 \mathrm{I}$ & 0.004 & 0.840 \\
$\begin{array}{l}\text { Elective admission length of } \\
\text { hospital stay (days) }\end{array}$ & 0.116 & 0.036 & 0.001 \\
$\begin{array}{l}\text { Secondary care outpatient } \\
\text { appointments }(\mathrm{n})^{\mathrm{f}}\end{array}$ & -0.069 & 0.019 & $<0.001$ \\
\hline
\end{tabular}

Notes: Multiple regression analysis adjusting for age, COPD severity, smoking status, and malnutrition risk (mean IMD score 15.74; SD II.06; SE 0.56; range 1.88-57.05). *Mean \pm SE (admission, appointments, or length of hospital stay [in days]) per patient per year after adjustment for the confounding variables listed in the table: ${ }^{\mathrm{a}} 0.615 \pm 0.072$; ${ }^{\mathrm{b}} 4.953 \pm 0.672$; ${ }^{\mathrm{c}} 0.365 \pm 0.055$; ${ }^{\mathrm{d}} 0.273 \pm 0.038$; ${ }^{\mathrm{e}} \mathrm{O} .180 \pm 0.383$; f $4.706 \pm 0.210$.

Abbreviation: IMD, Index of Multiple Deprivation.

that was significantly associated with elective admission duration (Table 2). The LOS per admission was variable for both emergency (7.9 [SD 8.8] days) and elective admissions (3.9 [SD 8.9] days), but only the duration of elective admission, after adjustment for confounders, was related to IMD ( $\beta$-coefficient $=0.324$, SE 0.096; $p=0.001)$. More-deprived individuals were less likely to participate in exercise rehabilitation programs (adjusted IMD score of participants 12.62, SE 1.43 vs nonparticipants 16.29 , SE $0.60 ; p=0.019)$. The total number of secondary care appointments, including those due to the exercise rehabilitation, was also inversely related to deprivation (Table 2).

The associations between health care use and individual deprivation domains were variable. Following adjustment for confounding variables, the annual number of emergency admissions per patient and the annual LOS per patient were significantly or borderline significantly $(p=0.050-0.099)$ related to employment ( $p=0.008$ and $p=0.063$, respectively), health deprivation/disability ( $p=0.053$ and $p=0.081$, respectively), education/skills/training $(p<0.001$ and $p=0.011$, respectively), and crime ( $p=0.041$ and $p=0.095$, respectively). While the number of elective admissions per patient was not significantly related to any of the individual deprivation domains, the duration of elective admissions was related to employment $(p<0.001)$, health deprivation/disability ( $p=0.039)$, education/skills/training $(p<0.001)$, living environment $(p=0.015)$, and crime $(p<0.076)$. Unlike the aforementioned relationships, which were positively related to the deprivation domains, secondary care appointments were inversely related to the following domains: health/ skills/training ( $p<0.001)$, crime $(p<0.001)$, living environment $(p<0.001)$, education/skills/training ( $p=0.004)$, and employment ( $p=0.056$ ). Similarly, participation in the exercise rehabilitation, which contributed to the secondary care appointments (Table 2), was also inversely related to several deprivation domains: health deprivation/disability $(p=0.017)$; crime $(p<0.001)$; and living environment $(p<0.001)$.

Individuals living in more-deprived areas had increased hospital inpatient costs, which outweighed the decreased secondary care outpatient costs (Table 3), reflecting the pattern in health care utilization (Table 2). The pattern and magnitude of the effects can also be appreciated by presenting the results after adjustment of health care use and costs for the same confounding variables, using deprivation as a fixed factor (after splitting the IMD scores into two halves), instead of as a continuous variable. For example, the moredeprived half had a greater total LOS in hospital from elective and emergency admissions, including those from the accident and emergency departments, and greater associated costs than the less-deprived half (9.6 [SE 1.3] days vs 5.6 [SE 1.4] days, $p=0.032$; and $£ 3,121$ [SE $£ 464$ ] vs $£ 2,034$ [SE $£ 469$ ]; $p=0.105$ ). The model involving IMD as a continuous variable, explained a small proportion of the total variability in cost (partial $\eta^{2}=0.056$ ), with deprivation explaining more of the variability (partial $\eta^{2}=0.029 ; p=0.001$ ) than smoking (partial $\eta^{2}=0.012 ; p=0.012$ ) and COPD severity (partial $\eta^{2}=0.012 ; p=0.012$ ).

\section{Mortality}

Mortality at 1 year was $10.6 \%$ and significantly related to deprivation (mortality in tertiles of increasing deprivation: 7.1\% vs 10.3 , vs 14.8 ; chi-squared [ $p$ for linear trend $=0.040]$ ).

Table 3 Influence of deprivation (IMD score) on annual secondary care health care costs $(\ell)(n=384)$

\begin{tabular}{llll}
\hline Health care use costs & $\beta$-coefficient* & SE & $\boldsymbol{p}^{\text {-value }}$ \\
\hline Emergency admissions $^{\mathrm{a}}$ & 57.16 & 25.6 & 0.026 \\
Elective admissions $^{\mathrm{b}}$ & 50.38 & 15.4 & $0.00 \mathrm{I}$ \\
Emergency and elective admissions $^{\mathrm{c}}$ & 107.54 & 29.78 & $<0.00 \mathrm{I}$ \\
Accident and emergency department $^{\mathrm{d}}$ & 0.67 & 0.570 & 0.243 \\
Secondary care outpatient $_{\text {appointments }}^{\mathrm{e}}$ & -6.88 & 1.93 & $<0.00 \mathrm{I}$ \\
Total costs $^{\mathrm{f}}$ & & & \\
\hline
\end{tabular}

Notes: Multiple regression analysis adjusting for age, COPD severity, malnutrition risk, and smoking status. The mean $( \pm \mathrm{SE})$ costs after adjustment for the listed confounding variables were as follows: ${ }^{a} \in 2,030.78 \pm 275.69$; ${ }^{b} \notin 5$ II.98 \pm 166.16 ;

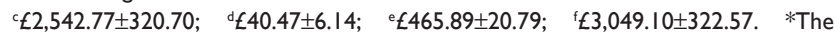
$\beta$-coefficient represents the annual increase in cost $(£, G B P)$ per patient per unit increase in IMD score (mean IMD score 15.74; SD I I.06; SE 0.56; 15.78 and range 1.88-57.05).

Abbreviations: IMD, Index of Multiple Deprivation; SE, standard error. 


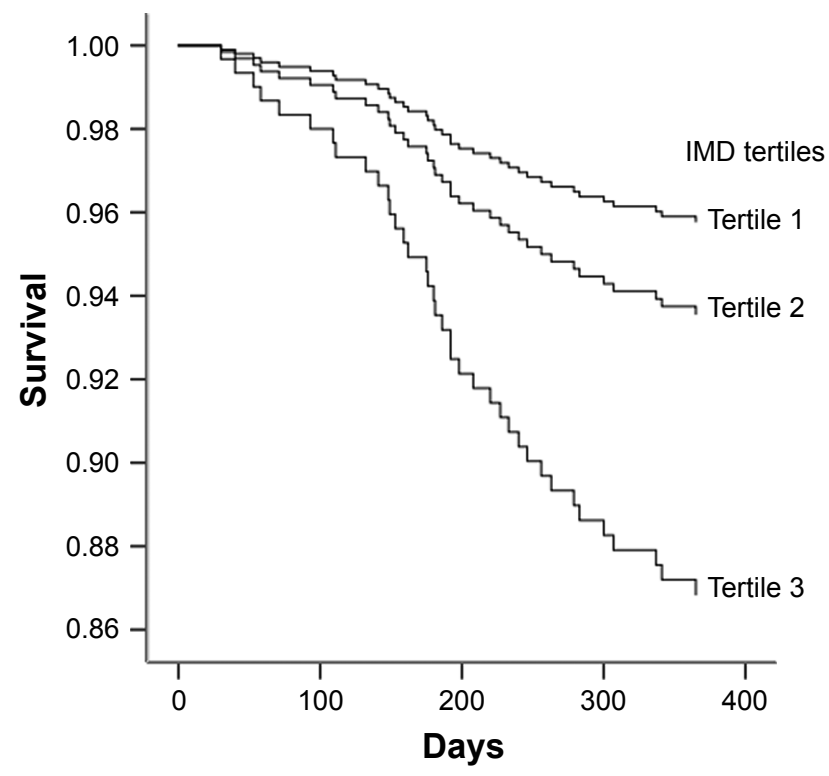

Figure I Cox regression analysis of I-year survival according to tertiles of IMD score, with adjustment for age, COPD disease severity, smoking status, and malnutrition risk.

Notes: $\mathrm{n}=384$; mean IMD score: 15.74 (SD II.06; SE 0.56; 15.78) and range: I.88-57.05; I= least deprived tertile, $3=$ most deprived tertile (overall $p=0.0 \mid 4$ ). With tertile 3 (most deprived) as referent, HR (in comparison with tertile I) $=0.306$ $95 \% \mathrm{Cl} 0.130-0.719$. $p=0.007$; and $\mathrm{HR}$ (in comparison with tertile 2 ) $=0.47 \mathrm{I}, 95 \% \mathrm{Cl}$ $0.226-0.984, p=0.045$.

Abbreviations: HR, hazard ratio; IMD, Index of Multiple Deprivation.

Using Cox regression analysis, deprivation was found to be a stronger predictor of mortality, after adjustment for confounders (HR 1.042, 95\% CI 1.015-1.070; $p=0.002$; refer Figure 1 for analysis using IMD tertiles). Age (HR $1.077,95 \%$ CI $1.033-1.121 ; p<0.001)$, malnutrition risk (HR 1.984, 95\% CI 1.010-3.894; $p=0.047$ ), and COPD severity (HR 1.984, 95\% CI 1.116-2.998; $p=0.017$ ) were also independent predictors of mortality at 1 year. In addition, mortality, adjusted for confounders, was related to individual deprivation domains with the following $p$-values: income ( $p=0.889$ ); employment ( $p=0.004)$; health deprivation/ disability $(p=0.004)$; education/skills/training $(p=0.004)$; barriers to housing ( $p=0.524)$, crime $(p=0.062)$; and living environment $(p=0.180)$.

\section{Discussion}

This study demonstrates the significant and independent association between social deprivation and health care use, health care costs, and mortality in outpatients with COPD. Deprivation was found to be associated with increased emergency hospitalization, increased LOS in hospital, and poorer survival. While deprivation was not associated with an increased rate of elective hospitalization, it was significantly associated with both a longer duration of elective hospital stay and associated costs. However, it is acknowledged that elective admissions are often not related to COPD but, often, to elective investigative or surgical procedures. Deprivation has previously been found to be associated with nonattendance at general practitioner (GP) appointments, ${ }^{15}$ and in the current study, a significant negative association existed between deprivation and both secondary care clinic attendance and participation in exercise rehabilitation. A previous study of COPD patients found deprivation and smoking prevalence to be significantly associated with hospitalization rates ${ }^{16}$ however, deprivation was assessed at the health district level rather than at the small area level (patient postcode). The present study is the first to explore the independent effect of deprivation in COPD patients in a smaller geographical area, examining the influence of deprivation closer to an individual level. An additional strength of the current study is that it attempted to explore the independent effect of deprivation, adjusting for confounders such as smoking status and malnutrition risk. The current findings are the first to report that deprivation is a significant and independent predictor of increased health care use, health care costs, and mortality when adjusting for age, COPD severity, smoking status, and malnutrition risk.

When exploring the impact of deprivation on health care use and clinical outcome, consideration of nutritional status and malnutrition risk is vital, as it has recently been shown to be independently associated with deprivation. ${ }^{11}$ In addition, malnutrition in patients with COPD has been found to present an economic and operational burden to hospitals, associated with increased health care use and costs, as well as poorer survival. ${ }^{17}$ Considering that deprivation is unlikely to be easily addressed in the short term, clinical interventions should be focused on the potentially modifiable risk factors, such as malnutrition and smoking status. Previous research suggests that socioeconomic differences in smoking cessation rates were not associated with the likelihood of attempting to stop smoking but were associated with success, suggesting that this may be due to greater nicotine dependence. ${ }^{18}$ Given the clustering of deprivation and malnutrition, as well as the fact that smoking cessation is often associated with weight gain, supporting individuals from deprived areas to successfully quit could be a useful multimodal intervention. ${ }^{19}$

Nutritional support in malnourished outpatients with stable COPD has been found to significantly improve nutritional intake and status, ${ }^{20}$ leading to improvements in functional capacity and quality of life. ${ }^{21}$ In addition, a large retrospective study involving hospital use of oral nutritional supplements in COPD patients was associated with reductions in length of admission, hospitalization costs, and readmission risk..$^{22}$ 
As financial deprivation has been found to be associated with poorer dietary intakes in COPD patients, ${ }^{23}$ future nutritional intervention studies should carefully consider deprivation. This study found a clustering of malnutrition, deprivation, and smoking status, with smoking being significantly associated with malnutrition risk and deprivation. A significant inverse relationship was found between age and deprivation, but it is not clear whether this is a unique observation relating to the current cohort residing in a small geographic area. Patients attending the smaller community hospital were significantly older, but this happened to be within a National Park and an area generally associated with affluence. Further investigation is also warranted around exercise rehabilitation and deprivation, as this study found that patients attending exercise programs were significantly more likely to reside in less-deprived areas. This could be due to differences in service provision and accessibility, as there is an increasing move for programs to be run in the community - rather than hospital - setting.

A limitation of the current study is that data were collected retrospectively with information unavailable on the reason for hospitalization or cause of mortality. It is acknowledged that this information, in addition to data on comorbidities, would have enriched analyses. Mortality in COPD patients is extremely complex as patients suffer from extrapulmonary comorbidities, such as coronary heart disease, malnutrition, and frailty, and they are also vulnerable to fatal outcomes such as pneumonia. All of these can result in the cause of death not being attributable directly to COPD. The study "TOwards a Revolution in COPD Health" (TORCH), involving 911 COPD patients, found respiratory disease to be the most common cause of death (35\%) and, of the deaths within that group, $27 \%$ specifically due to COPD and $8 \%$ due to pneumonia. ${ }^{24}$ Other common causes of death were lung cancer $(14 \%)$, other cancers $(7 \%)$, and stroke $(4 \%)$.

While IMD allows an overall estimation of deprivation, as well as its seven component domains (income, employment, health deprivation/disability, education/training/skills, barriers to housing, crime, and living environment), at the small area level, it does not directly assess an individual's deprivation per se but the likelihood that an individual experiences deprivation. Future research should attempt to assess indicators of deprivation at the individual level (eg, household income and educational attainment), and research exploring the prevalence of food insecurity experienced by patients with COPD as it relates to nutritional status is currently under way. More in-depth research is needed to assess causality, as deprivation association with the incidence of COPD may also be associated with greater progression of the disease, leading to disability, inability to continue work, and precipitate financial deprivation. Although a sample of $>400$ outpatients is reasonable to assess local associations, larger longitudinal population studies are needed in the future. It is plausible that the clustering of deprivation and poorer quality of life in COPD is partly driven by increased exacerbation rates requiring emergency hospitalization, with many individuals' health status never returning to the preexacerbation level. ${ }^{25}$ In a third of COPD patients, functional capacity and activities of daily living have been found to remain impaired 3 months after hospitalization. ${ }^{26}$ With exacerbation and hospitalization rates being strongly linked to deprivation, this raises the question whether more could be done for those COPD patients residing in deprived areas, as well as the possibility that more focused allocation of respiratory resources and expertise could hugely benefit the COPD population. ${ }^{27}$ Equitable access to health care is likely to have a large impact, with a previous study reporting that patients having easier access to see their preferred doctor was associated with a significant decrease in COPD mortality. ${ }^{28}$ It has been suggested that identifying these high-risk groups at a postcode level could allow targeted campaigns aiming to improve awareness and diagnosis of COPD, as well as being able to initiate targeted interventions to reduce the economic and operational burden of the disease. ${ }^{7}$ With deprivation associated with nonattendance at GP appointments ${ }^{15}$ and the current findings showing it is also related to nonattendance at hospital outpatient appointments, it is possible that patients living in more-deprived areas present to doctors later when symptomatic for infective exacerbations. It is unclear what influence deprivation has on adherence to prescribed medications in COPD (both maintenance therapy and medications to treat exacerbations), but it has been found to be associated with nonadherence in patients with Type 2 diabetes. ${ }^{29}$ To date, few studies have attempted to adjust for deprivation in their analyses; we hope that this research highlights the importance of deprivation, as well as its individual domains, as an important confounder. Pharmacological and nonpharmacological interventions should carefully consider deprivation as a potential barrier and tailor strategies accordingly.

\section{Conclusion}

Deprivation is significantly and independently associated with increased emergency health care use, health care costs, and poorer survival in COPD patients. Health inequalities are inextricably linked with social inequalities, but the routine assessment of deprivation within certain disease states such 
as COPD has the potential to inform targeted interventions. While deprivation is difficult to address, focused interventions on modifiable risk factors that cluster with deprivation, such as malnutrition risk and smoking status, could improve health outcomes in this particularly vulnerable patient group.

\section{Acknowledgment}

This study was funded through an unrestricted educational grant by Nutricia Ltd, UK, and was part of a nutritional intervention study, ClinicalTrials.gov identifier NCT00538200.

\section{Author contributions}

PFC, RJS, and ME designed the research; PFC and RJK coordinated and carried out the data collection across sites; PFC and ME analyzed the data; PFC, RJS, RJK, and ME wrote the manuscript; ME had primary responsibility for the final content. All authors contributed toward data analysis, drafting and critically revising the paper, gave final approval of the version to be published, and agree to be accountable for all aspects of the work.

\section{Disclosure}

PFC received an unrestricted educational grant from Nutricia Ltd. RJS is an employee of Nutricia Ltd. The authors report no other conflicts of interest in this work.

\section{References}

1. Simpson CR, Hippisley-Cox J, Sheikh A. Trends in the epidemiology of chronic obstructive pulmonary disease in England: a national study of 51804 patients. Br J Gen Pract. 2010;60(576):277-284.

2. Black DC. Inequalities in Health. London: Penguin; 1980.

3. Smith GD, Bartley M, Blane D. The Black Report on socioeconomic inequalities in health 10 years on. Br Med J. 1990;301:373-377.

4. Department of Health. The NHS Plan: A Plan for Investment, a Plan for Reform. London: Stationary Office; 2000.

5. Marmot M, Allen J, Goldblatt P, et al. Fair Society, healthy lives: The Marmot Review. London: The Marmot Review; 2010.

6. Prescott E, Lange P, Vestbo J. Socioeconomic status, lung function and admission to hospital for COPD: results from the Copenhagen City Heart Study. Eur Respir J. 1999;13(5):1109-1114.

7. COPD. British Lung Foundation. Invisible Lives: Chronic Obstructive Pulmonary Disease (COPD) - Finding the Missing Millions. London: COPD; 2007.

8. Noble M, McLennan D, Wilkinson K, Whitworth A, Barnes H, Dibden C. In: Government CaL, editor. The English Indices of Deprivation 2007. London: Communities and Local Government, UK; 2008.

9. Hawker JI, Olowokure B, Sufi F, Weinberg J, Gill N, Wilson RC. Social deprivation and hospital admission for respiratory infection: an ecological study. Respir Med. 2003;97(11):1219-1224.

10. Bottle A, Aylin P, Majeed A. Identifying patients at high risk of emergency hospital admissions: a logistic regression analysis. $J R$ Soc Med. 2006;99(8):406-414.
11. Collins PF, Elia M, Kurukulaaratchy RJ, Stratton RJ. The influence of deprivation on malnutrition risk in outpatients with chronic obstructive pulmonary disease (COPD). Clin Nutr. 2018;37(1):144-148.

12. Global Initiative for Chronic Obstructive Lung Disease. Pocket Guide to COPD Diagnosis, Management, and Prevention. 2017 Report. Available from: http://goldcopd.org/wp-content/uploads/2016/12/ wms-GOLD-2017-Pocket-Guide.pdf. Accessed March 30, 2018.

13. Elia M. The 'MUST' Report: Nutritional Screening for Adults - A Multidisciplinary Responsibility. Malnutrition Action Group (MAG). Redditch, UK: British Association of Parenteral and Enteral Nutrition (BAPEN); 2003.

14. Department of Health. NHS Reference Costs 2007-2008. London: Department of Health, UK; 2009:1-12.

15. Mercer SW, Watt GC. The inverse care law: clinical primary care encounters in deprived and affluent areas of Scotland. Ann Fam Med. 2007; 5(6):503-510.

16. Calderon-Larranaga A, Carney L, Soljak M, et al. Association of population and primary healthcare factors with hospital admission rates for chronic obstructive pulmonary disease in England: national cross-sectional study. Thorax. 2011;66(3):191-196.

17. Hoong JM, Ferguson M, Hukins C, Collins PF. Economic and operational burden associated with malnutrition in chronic obstructive pulmonary disease. Clin Nutr. 2017;36(4):1105-1109.

18. Kotz D, West R. Explaining the social gradient in smoking cessation: it's not in the trying, but in the succeeding. Tob Control. 2009;18(1): 43-46.

19. O’Hara P, Connett JE, Lee WW, Nides M, Murray R, Wise R. Early and late weight gain following smoking cessation in the Lung Health Study. Am J Epidemiol. 1998;148(9):821-830.

20. Collins PF, Stratton RJ, Elia M. Nutritional support in chronic obstructive pulmonary disease: a systematic review and meta-analysis. $\mathrm{Am} \mathrm{J}$ Clin Nutr. 2012;95(6):1385-1395.

21. Collins PF, Elia M, Stratton RJ. Nutritional support and functional capacity in chronic obstructive pulmonary disease: a systematic review and meta-analysis. Respirology. 2013;18(4):616-629.

22. Snider JT, Jena AB, Linthicum MT, et al. Effect of hospital use of oral nutritional supplementation on length of stay, hospital cost, and 30-day readmissions among Medicare patients with COPD. Chest. 2015;147(6):1477-1484.

23. Shalit N, Tierney A, Holland A, Miller B, Norris N, King S. Factors that influence dietary intake in adults with stable chronic obstructive pulmonary disease. Nutr Dietetics. 2016;73(5):455-462.

24. McGarvey LP, John M, Anderson JA, Zvarich M, Wise RA, Committee TCE. Ascertainment of cause-specific mortality in COPD: operations of the TORCH Clinical Endpoint Committee. Thorax. 2007;62(5): 411-415.

25. Seemungal TA, Donaldson GC, Bhowmik A, Jeffries DJ, Wedzicha JA. Time course and recovery of exacerbations in patients with chronic obstructive pulmonary disease. Am J Respir Crit Care Med. 2000;161(5): 1608-1613.

26. Peach H, Pathy MS. Follow-up study of disability among elderly patients discharged from hospital with exacerbations of chronic bronchitis. Thorax. 1981;36(8):585-589.

27. Partridge MR. Patients with COPD: do we fail them from beginning to end? Thorax. 2003;58(5):373-375.

28. Levene LS, Bankart J, Khunti K, Baker R. Association of primary care characteristics with variations in mortality rates in England: an observational study. PLoS One. 2012;7(10):e47800.

29. Donnan PT, MacDonald TM, Morris AD. Adherence to prescribed oral hypoglycaemic medication in a population of patients with Type 2 diabetes: a retrospective cohort study. Diabet Med. 2002;19(4):279-284. 
International Journal of COPD

Dovepress

\section{Publish your work in this journal}

The International Journal of COPD is an international, peer-reviewed journal of therapeutics and pharmacology focusing on concise rapid reporting of clinical studies and reviews in COPD. Special focus is given to the pathophysiological processes underlying the disease, intervention programs, patient focused education, and self management protocols.

This journal is indexed on PubMed Central, MedLine and CAS. The manuscript management system is completely online and includes a very quick and fair peer-review system, which is all easy to use. Visit http://www.dovepress.com/testimonials.php to read real quotes from published authors.

Submit your manuscript here: http://www.dovepress.com/international-journal-of-chronic-obstructive-pulmonary-disease-journal 